\title{
Exact solution and thermodynamics of the Hubbard model with infinite-range hopping
}

\author{
Peter van Dongen and Dieter Vollhardt \\ Institut für Theoretische Physik C, Technische Hochschule Aachen, D-5100 Aachen, Federal Republic of Germany
}

(Received 2 March 1989)

\begin{abstract}
The Hubbard model with unconstrained hopping of the particles on a lattice is solved exactly. It is shown that in this case the kinetic energy commutes with the interaction part, i.e., the model is essentially trivial. The thermodynamics is worked out explicitly. One finds that the results of the quasichemical approximation for the occupation probability of lattice sites are exact for this model. The ground state is insulating at half-filling and $U>0$ and is conducting otherwise.
\end{abstract}

The Hubbard model ${ }^{1}$ is the simplest model for interacting, itinerant electrons on a lattice. Nevertheless, except for dimension $d=1,{ }^{2}$ it is still much too difficult to be solved exactly. In this situation it is natural to investigate the model in simple, yet perhaps unrealistic, limits. ${ }^{3}$ The most common is that of an infinite-range (rather than zero-range) interaction, which leads to a trivial meanfield theory. Another limit is that of high dimensions, $d \rightarrow \infty,{ }^{4}$ which has only recently been introduced for the Hubbard model, ${ }^{5}$ and which may be expected to lead to new insight into the properties of the model in finite dimensions.

In this paper we discuss, and solve exactly, a version of the Hubbard model where the hopping of particles, rather than their interaction, has infinite range and occurs with equal probability. This is rather artificial in view of the physics underlying the derivation of the Hubbard model where hopping is assumed to be of short range (tight binding). Nevertheless, since the exact solution of this model has not been available so far, ${ }^{6}$ we will discuss it here.

The Hubbard model has the form ${ }^{1}$

$$
\begin{aligned}
& H=H_{0}+H_{I}-\mu \sum_{\mathbf{k}, \sigma} n_{\mathbf{k} \sigma}, \\
& H_{0}=\sum_{i j \sigma} t_{i j} c_{i \sigma}^{\dagger} c_{j \sigma}=\sum_{\mathbf{k}, \sigma} \varepsilon_{\mathbf{k}} n_{\mathbf{k} \sigma}, \\
& H_{I}=U \sum_{i} \hat{n}_{i \uparrow} \hat{n}_{i \downarrow}=U \sum_{\mathbf{k}} \rho_{\mathbf{k} \uparrow} \rho_{-\mathbf{k} \downarrow},
\end{aligned}
$$

where the kinetic energy $H_{0}$ and the on-site interaction $H_{I}$ have been expressed both in position and momentum representation. Here $c_{i \sigma}^{\dagger}\left(a_{\mathrm{k} \sigma}^{\dagger}\right)$ creates a particle with spin $\sigma$ at site $i$ (with momentum $\mathbf{k}$ ), respectively, and

$$
\rho_{\mathbf{k} \sigma}=L^{-1 / 2} \sum_{\mathbf{q}} a_{\mathbf{q} \sigma}^{\dagger} a_{\mathbf{q}+\mathbf{k}, \sigma}
$$

is the Fourier component of the local density. We assume $n_{\uparrow}=n_{\downarrow}=n / 2$ with $\mu$ as the common chemical potential. While $H_{0}$ is diagonal in $\mathbf{k}$ space, $H_{I}$ is diagonal in position space since it is given by the number operator of doubly occupied sites $D=\sum_{i} D_{i}$, with $D_{i}=n_{i \uparrow} n_{i \downarrow}$. We now assume the hopping to have infinite range, with $t_{i j} \equiv-t$ for all $i, j$. In this case the actual lattice structure, as well as the dimensionality of the system, becomes unimportant. From (1b) it follows that

$$
\varepsilon_{\mathbf{k}}=-t L \delta_{\mathbf{k}, 0}
$$

where $L$ is the number of lattice sites. Clearly, the operator

$$
A_{\sigma} \equiv L^{-1 / 2} \sum_{i} c_{i \sigma}=a_{\mathrm{k}=0, \sigma}
$$

still obeys Fermi statistics with $A_{\sigma}^{\dagger} A_{\sigma}=n_{\mathrm{k}=0, \sigma}$. Note, that - in contrast to naive expectation - the hopping constant must not be scaled with $L$ for the average kinetic energy

$$
\bar{\varepsilon}_{0}=\sum_{k<k_{F}, \sigma} \varepsilon_{\mathbf{k}}=-2 t L
$$

to remain extensive. Equation (2) implies that the two particles in the state $\mathbf{k}=0$ carry the entire kinetic energy of the system, while states with $\mathbf{k} \neq 0$ do not contribute and are degenerate. The question is then whether $\mathbf{k}=0$ contributes significantly to the interaction terms at all. Below we will show that this is not the case, i.e., that the expectation value of $\left[H_{0}, H_{I}\right]$ vanishes in the thermodynamic limit, such that $H_{0}, H_{I}$ may be diagonalized separately.

To calculate the expectation value of $H$, (1a), with (2), we introduce the partition function via a time-ordered exponential $^{7}$

$$
\begin{aligned}
Z(t, U) & =\operatorname{tr}\left[T \exp \left[-\int_{0}^{\beta} d \tau H(\tau)\right]\right] \\
& =Z_{0}\left\langle\exp \left[-\int_{0}^{\beta} d \tau H_{I}(\tau)\right]\right\rangle_{0} .
\end{aligned}
$$

Here $H(\tau)$ is given by (1a) with $n_{\mathbf{k} \sigma}=n_{\mathbf{k} \sigma}(\tau)$ and $H_{I}=H_{I}(\tau)$, and \langle\rangle$_{0}$ is the thermal average in terms of $Z_{0}=Z(t, 0)$. In principle $Z$ can be calculated from (3b) in a diagrammatic expansion, using Wick's theorem. The grand canonical potential $\Omega=-\beta^{-1} \ln Z$ follows as $\Omega=\Omega_{0}-\beta^{-1} W_{t}(\beta, U)$, where $\Omega_{0}=-\beta^{-1} \ln Z_{0}$ and

$$
W_{t}(\beta, U) \equiv\left\langle\exp \left(-\int_{0}^{\beta} d \tau H_{I}(\tau)\right]\right\rangle_{0}^{c}
$$

is obtained from \langle\rangle$_{0}$ by retaining only connected diagrams. The subscript $t$ indicates the explicit dependence of $W_{t}$ on the hopping constant. The propagators in these 
diagrams are calculated with respect to $Z_{0}$ and have the form

$$
\left\langle T a_{\mathbf{k} \sigma}(\tau) a_{\mathbf{k}^{\prime} \sigma^{\prime}}^{\dagger}\left(\tau^{\prime}\right)\right\rangle_{0}=\delta_{\mathbf{k k}^{\prime}} \delta_{\sigma \sigma^{\prime}} G_{\mathbf{k} \sigma}\left(\tau-\tau^{\prime}\right),
$$

where

$$
G_{\mathbf{k} \sigma}(\tau)= \begin{cases}e^{-\left(\varepsilon_{\mathbf{k}}-\mu\right) \tau}\left(1-f_{\mathbf{k} \sigma}\right) & (\tau>0), \\ -e^{-\left(\varepsilon_{\mathbf{k}}-\mu\right) \tau} f_{\mathbf{k} \sigma} & (\tau \leq 0)\end{cases}
$$

with $f_{\mathbf{k} \sigma}=\left\{\exp \left[\beta\left(\varepsilon_{\mathbf{k}}-\mu\right)\right]+1\right\}^{-1}$ as the Fermi function. Equation (6) implies that $G_{\mathbf{k} \sigma}$ is independent of $\mathbf{k}$ for all $\mathbf{k} \neq 0$, while for $\mathbf{k}=0$,

$$
G_{\mathbf{k}=0, \sigma}(\tau)=0, \quad \tau<0 \text { or } 0<\tau<\beta
$$

and $G_{0 \sigma}(0)=-G_{0 \sigma}(\beta)=-1$. If one now calculates $W_{t}$, (4), in perturbation theory, one has

$W_{t}=\sum_{m=1}^{\infty} \frac{(-1)^{m}}{m !} \int_{0}^{\beta} d \tau_{1} \cdots \int_{0}^{\beta} d \tau_{m}\left\langle H_{I}\left(\tau_{1}\right) \cdots H_{I}\left(\tau_{m}\right)\right\rangle_{0}^{c}$

Clearly, $W_{t}$ is completely determined by contributions from $\mathbf{k} \neq 0$ since the propagator for $\mathbf{k}=0$ vanishes according to (7). One may therefore just as well put $t=0$ in (4) but nevertheless include the momentum $\mathbf{k}=0$ in the $\mathbf{k}$ sums, since the error introduced thereby is only of relative order $1 / L$ :

$$
W_{t}=W_{t=0}+O(1 / L) .
$$

In the thermodynamic limit $W_{t}$ is therefore seen to be independent of $t$. Hence the partition function $Z(t, U)$ in (3a) may be expressed in terms of the partition function for $t=0$ as

$$
Z(t, U)=Z(0, U) \exp (2 \beta t L)
$$

with

$$
Z(0, U)=\sum_{\left\{n_{i \sigma}\right\}} \exp \left[\beta \mu \sum_{i, \sigma} n_{i \sigma}-\beta U \sum_{i} D_{i}\right]
$$

The Hamiltonian in (9) is site diagonal, such that

$$
Z(0, U)=\left(1+2 z+z^{2} e^{-\beta U}\right)^{L},
$$

where $z=e^{\beta \mu}$ is the fugacity. In this way one finally finds

$$
\Omega=L\left[-2 t-\beta^{-1} \ln \left(1+2 z+z^{2} e^{-\beta U}\right)\right],
$$

which is exact in the thermodynamic limit. The contributions from $H_{0}$ and $H_{I}$ are seen to decouple completely, since $\mathbf{k}=0$ (the only relevant state for the kinetic energy) is irrelevant for the interaction energy in the thermodynamic limit. Hence, both parts of the Hamiltonian may be diagonalized individually. In this sense the model is essentially trivial.

All thermodynamic quantities of the model are determined by (11). In particular, the chemical potential $\mu$ is obtained from the density

$$
n=N / L=-L^{-1}(\partial \Omega / \partial \mu)_{\beta}
$$

as

$$
\begin{gathered}
\mu=U+k_{B} T \ln \left(\left\{\left[(1-n)^{2}+n(2-n) e^{-\beta U}\right]^{1 / 2}\right.\right. \\
-(1-n)\} /(2-n)),
\end{gathered}
$$

which for $n=1$ reduces to $\mu=U / 2$. For $n \leq 1$ the density of doubly occupied sites, $\bar{d}=D / L=\left\langle H_{I}\right\rangle / U$, is found as $^{8}$

$$
\bar{d}(n, \beta U)=\frac{\left[(n-1)^{2}+n(2-n) e^{-\beta U}\right]^{1 / 2}-1+n\left(1-e^{-\beta U}\right)}{2\left(1-e^{-\beta U}\right)} .
$$

In particular, for $n=1$

$$
\bar{d}=\frac{1}{2} \frac{1}{1+e^{\beta U / 2}} .
$$

The parameter $\bar{d}$ only depends on temperature and interaction via the single parameter $\beta U$. Equation (13) may also be written as

$$
\frac{\bar{d}(1-n+\bar{d})}{(n / 2-\bar{d})^{2}}=e^{-\beta U} .
$$

This expression has the form known from the law of mass action, with the Boltzmann factor $e^{-\beta U}$ regulating the equilibrium between the total concentration of doubly occupied sites $(\bar{d})$ and empty sites $(1-n+\bar{d})$ relative to that of the singly occupied sites $\left[(n / 2-\bar{d})^{2}\right]$. Equation (15) is typical for a result obtained within the "quasichemical approximation" in the theory of mixtures. " It is interesting to note that (15) is also identical to the result of the Gutzwiller approximation ${ }^{10}$ for the conventional Hubbard model, if $e^{-\beta U}$ is replaced by $g^{2}$, where $g$ is a variational parameter entering the trial wave function used in the approach. Indeed, this approximation is precisely of the quasichemical type and is known to yield the correct result for expectation values of the ground-state energy, etc., in terms of the trial wave function in $d=\infty .{ }^{5}$ Equation (14) had already been obtained by Seiler et al. ${ }^{11}$ within a phenomenological extension of the Gutzwiller approximation ${ }^{10}$ to finite temperatures. In the model discussed here (15) is exact; hence, the quasichemical approximation, with equilibrium constant $e^{-\beta U}$, is found to be exact in this case. This is not surprising since hopping is completely unconstrained in the model and every site can be reached without restriction.

At $T=0$ (13) implies that $\bar{d}(U)=0$ for $n \leq 1$ and $\bar{d}(U)=n-1$ for $n>1$, independent of the strength of $U$, i.e., the unrestricted hopping allows the system to assume a ground state with the least possible number of doubly occupied sites. Hence, $\bar{d}(U)$ has a kink at $n=1$. On the other hand, for $T>0$ one obtains

$$
\bar{d}(U) \simeq\left[\frac{n}{2}\right]^{2}-\left[\frac{n(2-n)}{4}\right]^{2} \beta U, \quad \beta U \rightarrow 0
$$


and

$$
\bar{d}(U)=\left\{\begin{array}{l}
\frac{n^{2}}{4(1-n)} e^{-\beta U}, \quad n<1, \quad \beta U \rightarrow \infty, \\
\frac{1}{2} e^{-\beta U / 2}, \quad n=1, \quad \beta U \rightarrow \infty .
\end{array}\right.
$$

Hence for $T>0, \bar{d}(U)$ has the form one should expect it to have in the conventional Hubbard model, namely $\bar{d}(0)=(n / 2)^{2}$ and an exponential decrease for $U \rightarrow \infty$. Note that the half-filled case $(n=1)$ leads to a different dependence on $U$ than that for $n<1$.

The pressure, $P=-\Omega / L$, of the model is given by

$$
P= \begin{cases}2 t+n k_{B} T, \quad n \rightarrow 0, & T \text { fixed } \\ \ln \left(1-\frac{n}{2}\right)^{-2} k_{B} T, & n \rightarrow 2, \quad T \text { fixed }\end{cases}
$$

and by

$$
P=2 t+\frac{1}{2} U+\ln 2\left(k_{B} T\right), \quad n=1, \quad T \rightarrow 0 .
$$

Hence, $P$ remains finite even for $n \rightarrow 0$. This unrealistic dependence is caused by the two particles at $\mathbf{k}=0$, which produce the complete extensive kinetic energy.

The entropy is given by

$$
S=k_{B} L\left[\beta U \bar{d}-\beta \mu n+\ln \left(1+2 z+z^{2} e^{-\beta U}\right)\right] .
$$

It approaches a finite value both for $T \rightarrow 0$ (due to the large degeneracy of the ground state) and for $T \rightarrow \infty$ (due to the finite number of possible states on a lattice). From the internal energy

$$
E=\Omega+T S+\mu N=L(-2 t+U \bar{d})
$$

the specific heat follows as

$$
C_{v}=-k_{B} L U \beta^{2}\left(\frac{\partial \bar{d}}{\partial \beta}\right]_{n} .
$$

At low temperatures $(\beta U \rightarrow \infty)$ it approaches zero exponentially fast

$$
C_{v}=\left\{\begin{array}{l}
\frac{n^{2}}{4(1-n)} k_{B} L(\beta U)^{2} e^{-\beta U} \quad(n<1), \\
\frac{1}{4} k_{B} L(\beta U)^{2} e^{-\beta U / 2} \quad(n=1),
\end{array}\right.
$$

while at high temperatures $(\beta U \rightarrow 0)$ it vanishes as $T^{-2}$ according to

$$
C_{v}=\left[\frac{1}{4} n(2-n)\right]^{2} k_{B} L(\beta U)^{2} .
$$

Finally, we remark on the conduction properties of the model. Introducing independent chemical potentials $\mu_{\sigma}$, i.e., particle numbers $N_{\sigma}$, for the spins, the criterion of Mattis ${ }^{12}$ and Lieb and $\mathrm{Wu}^{2}$ states that the ground state is insulating if the difference of the energies $E\left(N_{\uparrow}, N_{\downarrow}\right)$

$$
\begin{aligned}
\Delta \mu \equiv \lim _{T \rightarrow 0}\left[E\left[\frac{N}{2}+1, \frac{N}{2}\right)+E\left(\frac{N}{2}-1, \frac{N}{2}\right)\right. \\
\left.-2 E\left[\frac{N}{2}, \frac{N}{2}\right)\right]
\end{aligned}
$$

is positive, and is conducting for $\Delta \mu=0$. In the model investigated here $\Delta \mu$ is entirely determined by $\bar{d}\left(N_{\uparrow}, N_{\downarrow}\right)$

$$
\begin{aligned}
\Delta \mu=U L \lim _{T \rightarrow 0} & {\left[\bar{d}\left[\frac{N}{2}, \frac{N}{2}-1\right]+\bar{d}\left(\frac{N}{2}-2, \frac{N}{2}-1\right]\right.} \\
& \left.-2 \bar{d}\left(\frac{N}{2}-1, \frac{N}{2}-1\right]\right] .
\end{aligned}
$$

Since $\bar{d}$ has a kink at half-filling [cf. the discussion above Eq. (16a)] it follows that in this case $\Delta \mu>0$ (insulating ground state), while $\Delta \mu=0$ (conducting ground state) for all other fillings. It is interesting to note that the exact ground state of the ordinary Hubbard model in $d=1$ is insulating too at half-filling. ${ }^{2}$ However, in the latter case the origin of the insulating behavior is different, being a consequence of the antiferromagnetic correlations by the perfect nesting property of the lattice for $U>0$.

In summary, we have presented the exact solution and thermodynamics of the Hubbard model in the case where hopping of particles may occur to any lattice site with equal transition rate. In this situation the two terms of the Hamiltonian commute, i.e., do not compete with each other, and can thus be diagonalized independently. This is true also for general interactions and kinetic energies, as long as $\varepsilon_{\mathbf{k}} \neq 0$ only for a nonextensive number of $\mathbf{k}$ states. Although the model is, therefore, essentially a trivial one, its solution allows for some interesting conclusions: (i) the result for the interaction energy, i.e., the number of doubly occupied sites $\bar{d} L$, has a form known from the law of mass action with equilibrium constant $e^{-\beta U}$, (ii) in the ground state $\bar{d}(U)=0$ for $n \leq 1$ and arbitrary $U$, (iii) at any $T>0$ with $T$ fixed $\bar{d}(U)$ has a form that one expects from the conventional Hubbard model, (iv) the ground state is insulating in the half-filled case for all $U>0$ and is conducting for other fillings.

\section{ACKNOWLEDGMENTS}

One of us (D.V.) thanks L. Stodolsky, C. Castellani, F. D. M. Haldane, and A. E. Ruckenstein for useful discussions. We are also grateful to F. Gebhard and W. Metzner for many helpful discussions. This work was supported in part by the Sonderforschungsbereich (SFB) 341 of the Deutsche Forschungsgemeinschaft.
${ }^{1}$ M. C. Gutzwiller, Phys. Rev. Lett. 10, 159 (1963); J. Hubbard, Proc. R. Soc. London, Ser. A 276, 238 (1963); J. Kanamori, Prog. Theor. Phys. 30, 275 (1963).

${ }^{2}$ E.H. Lieb and F. Y. Wu, Phys. Rev. Lett. 20, 1445 (1968).
${ }^{3}$ For an early review of the Hubbard model, see M. Cyrot, Physica $91 \mathrm{~B}, 141$ (1977).

${ }^{4}$ In this limit and for $U \rightarrow \infty$, where the Hubbard model is equivalent to the Heisenberg model, the Neel state is known 
to be the exact ground state [T. Kennedy, E. H. Lieb, and B. S. Shastry, Phys. Rev. Lett. 61, 2582 (1988)].

${ }^{5}$ W. Metzner and D. Vollhardt, Phys. Rev. Lett. 62, 324 (1989).

${ }^{6}$ The model was considered previously by one of us (D.V.), who also discussed it with participants of the Aspen workshop on "Heavy Fermions and Valence Fluctuations" in 1985; at that occasion F. D. M. Haldane mentioned that he had been looking into the same model independently. At the time no conclusion was reached and no published work resulted. The model itself seems to have been discussed first by J. D. Patterson [Phys. Rev. B 6, 1041 (1972)] who investigated it numerically for very small systems. We are grateful to K. A. Penson for this information. However, such an approach cannot lead to significant insight since, in view of the importance of the $\mathbf{k}=0$ state, a study of small systems is clearly inadequate.

${ }^{7}$ See, for example, J. W. Negele and H. Orland, Quantum Many-Particle Systems (Addison-Wesley, Menlo Park, 1988).

${ }^{8}$ The case $1<n \leq 2$ is obtained by particle-hole symmetry as $\bar{d}(2-n)=\bar{d}(n)+1-n$.

${ }^{9}$ E. A. Guggenheim, Mixtures (Oxford University, New York, 1952), p. 38.

${ }^{10}$ M. C. Gutzwiller, Phys. Rev. A 137, 1726 (1965); for a simple exposition see D. Vollhardt, Rev. Mod. Phys. 56, 99 (1984).

${ }^{11}$ K. Seiler, C. Gros, T. M. Rice, K. Ueda, and D. Vollhardt, J. Low Temp. Phys. 64, 195 (1986).

${ }^{12}$ D. Mattis (unpublished) (cited in Ref. 2). 